\title{
The Effects of Bilateral Arm Training on Reaching Performance and Activities of Daily Living of Stroke Patients
}

\author{
Nam-Hae Jung ${ }^{1)}$, Kyeong-Mi Kim ${ }^{2)}$, Jae-Seop OH ${ }^{3)}$, Moonyoung Chang ${ }^{2}{ }^{*}$ \\ 1) Department of Occupational Therapy, Bongseng Memorial Hospital \\ 2) Department of Occupational Therapy, College of Biomedical Science and Engineering, Inje \\ University: 197 Inje Street, Gimhae, Gyeongsangnam-do 621-749, Republic of Korea. \\ TEL: +82 55-320-3685, FAX: +82 55-326-4885 \\ 3) Department of Physical Therapy, College of Biomedical Science and Engineering, Inje University
}

\begin{abstract}
Purpose] To examine effects of Bilateral Arm Training (BAT) on unilateral and bilateral reaching performance and the performance of activities of daily living (ADL) of stroke patients. [Methods] Fifteen participants received 4 weeks of BAT. Unilateral and bilateral reaching were measured using 3D motion analysis. Performance of and client satisfaction with ADL were assessed by the Canadian Occupational Performance Measure. The amount and quality of use of the affected upper limb were assessed by the Motor Activity Log. [Results] BAT showed statistically significant improvements in the average and peak velocities during reaching performance, and the amount and quality of use of the affected upper limb. There were statistically significant improvements in the performance of and client satisfaction with ADL. However, the result did not show a statistically significant difference in the trajectory ratio during reaching performance. [Conclusion] BAT was significantly effective at improving the velocity of reaching performance and the performance of ADL by stroke patients. In the future, studies should investigate the effects of the duration and intensity of training, and a variety of BAT protocols need to be developed. Key words: Activities of daily living (ADL), Stroke, Upper extremity
\end{abstract}

(This article was submitted Oct. 24, 2012, and was accepted Dec. 4, 2012)

\section{INTRODUCTION}

Upper extremity dysfunction is a frequent symptom after stroke ${ }^{1)}$, and it is related to performance in Activities of Daily Living $(\mathrm{ADL})^{2)}$. Various therapeutic interventions for improvement of the upper extremity focus on the unilateral upper extremity function. It is difficult for stroke patients to perform ADL requiring bilateral hand use ${ }^{3)}$. So, to improve bilateral upper extremity function, Bilateral Arm Training (BAT) must be implemented.

A basic assumption of BAT is that symmetrical bilateral movements activate similar neural networks in both hemispheres when homologous muscles are simultaneously activated ${ }^{4}$. Although the upper extremity is moved unilaterally, inhibition of the ipsilateral hemisphere and interhemispheric inhibition occur to prevent mirror movement of the opposite upper extremity ${ }^{5}$. When both upper extremities are moved for symmetrical task performance, intra-cortical inhibition is reduced and both hemispheres are activated ${ }^{6}$. Recently, studies involving kinetic analysis of the upper extremity have been increasing ${ }^{7}$. However, most studies in Korea have reported the composite scores of standardized tests rather than determining how the motor control of arm movements has changed. Therefore, the pur-

*To whom correspondence should be addressed. E-mail: myot@inje. ac.kr pose of this study was to investigate the effects of BAT on the reaching performance of chronic stroke patients using $3 \mathrm{D}$ motion analysis, and to investigate the effects of BAT on performance of ADL, the amount and quality of affected upper extremity use along with performance of and client satisfaction with ADL were assessed.

\section{SUBJECTS AND METHODS}

Fifteen hemiplegic patients who had no problem with their cognition and had voluntary reaching movement on the affected side were recruited for this study. The Brunnstrom stage of the upper extremities of all participants were 3 or above (Table 1). All participants read and signed an informed consent form, approved by Inje University Ethics Committee for Human Investigations prior to their participation.

A BAT protocol was constructed for this study and its content validity was verified using a questionnaire by an expert panel. The procedures were as follows. The operational definition of BAT is when both arms, including the shoulders, elbows, wrists and hands, symmetrically perform activities including reaching, grasping and carrying at the same time. Various upper extremity rehabilitation protocols using tasks in advanced research were designed according to the operational definition. The expert panel involved two professors and two occupational therapists. 
Fifteen participants received BAT in 30 minute treatment sessions, 5 times a week for 4 weeks. The BAT protocol was composed of a warming-up stage, the main stage and a cooling-down stage. The joints and muscles of both arms were stretched for 5 minutes in the warm-up stage. In the main stage, intensive BAT was performed for 20 minutes. There were toweling, sanding, simulation of drinking, carrying blocks, stacking cones and pegboard tasks in the main stage. After the main stage, the joints and muscles of both arms were stretched for 5 minutes in the cooling-down stage, just as in the warm up stage.

At pre- and post-intervention, reaching performance was assessed by 3D motion analysis, and ADL was assessed by the Canadian Occupational Performance Measure (COPM) and the Motor Activity Log (MAL).

Kinematic data for arm movement during unilateral reaching and bilateral reaching movements were collected using a CMS 10 (Zebris Medical GmbH, Germany). Three markers were attached to the distal interphalangeal joint, the metacarpophalangeal joint of the index finger, and the dorsal side of the wrist. The measured tasks were unilateral reaching with the affected upper extremity and bilateral reaching. Both tasks were performed three times. This study analyzed the average velocity, the peak velocity and the trajectory ratio. The trajectory ratio was defined as the ratio between the actual hand travel path and a straight line from movement onset to movement completion. The trajectory ratio was calculated using the MatLab program version 2008a (The MathWorks Inc, 2008).

The MAL is a scripted, structured interview which assesses the amount of use and quality of movement of the hemiparetic arm in thirty ADLs using a six-point, 0-5, Likert scale. The better the quality of movement and amount of use, the higher the score. The internal consistency has been reported as $0.88: 0.95$, the interexaminer reliability as 0.90 , and the test-retest reliability as $0.94^{8)}$.

The COPM is a semi-structured assessment which evaluates the three areas of self-care, work, and leisure activities. The performance and satisfaction of five activities which the clients select by importance are self-assessed. Importance, performance and satisfaction are assessed on a tenpoint Likert scale (1-10). A difference of two or more points between pre-intervention and post-intervention assessment scores is considered clinically significant ${ }^{9}$.

Statistical analysis in this study was done using SPSS version 18.0. The general characteristics of the participants were analyzed using descriptive statistics and the differences between the pre- and post-intervention assessment scores were analyzed using the paired t-test. The significance level was chosen as 0.05 .

\section{RESULTS}

The average velocity for unilateral reaching significantly increased from $293.2 \mathrm{~mm} / \mathrm{s}$ to $512.2 \mathrm{~mm} / \mathrm{s}(\mathrm{p}<0.05)$. The average velocity for bilateral reaching significantly increased from $318.2 \mathrm{~mm} / \mathrm{s}$ to $589.5 \mathrm{~mm} / \mathrm{s}(\mathrm{p}<0.05)$. The peak velocity for unilateral reaching significantly increased from $458.4 \mathrm{~mm} / \mathrm{s}$ to $582.0 \mathrm{~mm} / \mathrm{s}(\mathrm{p}<0.05)$. The peak velocity for
Table 1. General characteristics of subjects

\begin{tabular}{lcc}
\hline Characteristic & & N (\%) \\
\hline \multirow{2}{*}{ Gender } & Male & $11(73)$ \\
& Female & $4(27)$ \\
Age (years) & $30-39$ & $1(6)$ \\
& $40-49$ & $1(6)$ \\
Cause & $50-59$ & $7(47)$ \\
& 60 and above & $6(41)$ \\
Affected side & Hemorrhage & $9(60)$ \\
& Infarction & $6(40)$ \\
& Right & $10(67)$ \\
Brunnstrom stage & Left & $5(33)$ \\
& 1 & $0(0)$ \\
& 2 & $0(0)$ \\
Time since stroke & 3 & $4(27)$ \\
(months) & 4 & $5(33)$ \\
& 5 & $4(27)$ \\
& $6-13$ & $2(13)$ \\
& $14-20$ & $7(47)$ \\
& $21-27$ & $6(40)$ \\
\end{tabular}

bilateral reaching significantly increased from $501.5 \mathrm{~mm} / \mathrm{s}$ to $659.5 \mathrm{~mm} / \mathrm{s}(\mathrm{p}<0.05)$. The trajectory ratio for unilateral reaching improved from 1.38 to 1.37 , and for bilateral reaching improved from 1.36 to 1.26 ( $\mathrm{p}>0.05)$, but these differences were not statistically significant. Details are shown in Table 2.

Performance of COPM significantly improved from 2.27 to $3.43(\mathrm{p}<0.05)$. The satisfaction of COPM significantly improved from 2.23 to $3.87(\mathrm{p}<0.05)$. Details are shown in Table 3.

Results of the analysis of the amount of affected upper limb use, showed a statistically significant improvement from 23.1 to $31.6(\mathrm{p}<0.05)$. The quality of affected upper limb movement significantly improved from 25.7 to 33.5 $(p<0.05)$. Details are shown in Table 3.

\section{DISCUSSION}

The purpose of this study was to examine the effects of BAT on unilateral and bilateral reaching and performance of ADL of stroke patients. A BAT protocol which had been verified by a professional panel was implemented in this study. This study discussed the operational definition of BAT. It is important that this protocol which was verified for content validity is easy to implement in a clinical environment.

Recently, research concerning motor capacity of stroke patients has been conducted using more objective and scientific ways. One study used a 3D motion analysis system to kinematically analyze the performance ${ }^{10)}$. This study analyzed the average velocity, peak velocity and trajectory ratio which are important spatial and temporal parameters of upper extremity motions. Some studies which have investigated the effect of BAT on reaching, using 3D motion 
Table 2. Comparison of average velocity, peak velocity and trajectory ratio

\begin{tabular}{lccc}
\hline & & Pre-intervention & Post-intervention \\
\hline $\begin{array}{l}\text { Average } \\
\begin{array}{l}\text { Velocity } \\
(\mathrm{mm} / \mathrm{s})\end{array}\end{array}$ & Unilateral* & $293.2 \pm 40.1$ & $512.2 \pm 70.1$ \\
\hline $\begin{array}{l}\text { Peak } \\
\begin{array}{l}\text { Velocity } \\
(\mathrm{mm} / \mathrm{s})\end{array}\end{array}$ & Unilateral*a & $318.2 \pm 37.5$ & $589.5 \pm 73.3$ \\
\hline $\begin{array}{l}\text { Trajectory } \\
\text { ratio }\end{array}$ & Bilateral** & $458.4 \pm 55.1$ & $582.0 \pm 55.7$ \\
\hline$* \mathrm{p}<0.05, * * \mathrm{p}<0.01$ & Bilateral & $1.4 \pm 0.06$ & $1.368 \pm 0.04$ \\
& $1.36 \pm 0.05$ & $1.28 \pm 0.05$ \\
\hline
\end{tabular}

Table 3. Comparison of pre and post-intervention scores of COPM and MAL

\begin{tabular}{cccc}
\hline & & Pre-intervention & Post-intervention \\
\hline \multirow{2}{*}{ COPM } & Performance** & $2.27 \pm 0.18$ & $3.43 \pm 0.29$ \\
& Satisfaction** $^{*}$ MAL & $2.23 \pm 0.21$ & $3.87 \pm 0.34$ \\
\hline \multirow{2}{*}{ Amount of Use** } & $23.1 \pm 2.6$ & $31.7 \pm 3.2$ \\
& Quality of Movement** & $25.7 \pm 4.3$ & $33.5 \pm 4.3$ \\
\hline \multirow{2}{*}{$\mathrm{m}<0.01$} & & &
\end{tabular}

analysis systems, have compared unilateral and bilateral reaching. McCombe Waller et al. ${ }^{11)}$ reported that BAT significantly improved movement unit, movement time and peak acceleration of the affected side, and movement time, peak acceleration, and the trajectory ratio of the unaffected side in bilateral reaching compared to unilateral reaching. Moreover, Cauraugh et al. ${ }^{12)}$ found that BAT significantly improved movement unit, peak velocity and deceleration time in bilateral reaching. Some studies reported only the effects of BAT on unilateral reaching. Summers et al. ${ }^{13)}$ found a significant improvement in the elbow angle. Senesac et al. ${ }^{10)}$ reported a significant improvement of hand path curvature, smoothness of the curve, and peak velocity. The present study examined both bilateral and unilateral reaching. The average velocity and peak velocity significantly improved after BAT, but the trajectory ratio did not. The effect of BAT on movement velocity was reported by Chang et al. ${ }^{14)}$. They reported that the trajectory ratio improved only in bilateral reaching, not in unilateral reaching after BAT. Actually, temporal parameters like velocity are easier to influence than spatial parameters like the trajectory ratio $^{15}$. It seems that the protocol used in the present study had limitations with regard to the duration and intensity of intervention.

Quality of life means that the individual is personally aware about goals, expectations, norms, and interests in their cultural environment and values. Quality of life for patients is related to satisfaction with life. So, ADL was assessed by COPM measuring the performance, and satisfaction of the client with ADL; and MAL was used to assess the amount and quality of the affected upper limb use. Wu et al. ${ }^{16)}$ reported three out of four subjects showed an improved score of MAL after BAT, but the difference was not statistically significant. The MAL results of the present study indicate that BAT improved the performance of ADL requiring bilateral hand use. The result of COPM showed only statistically significant effects, not clinical differences. We consider that this is because of the short intervention duration and standardized protocol.

In the future, studies of BAT should use assessments of bilateral function. Moreover, there is a need to develop an assessment of bilateral function during the performance of ADL. The number of participants in this study was insufficient to generalize the result. This study did not conduct a follow-up test. The one group pretest-posttest design of this study provides weak evidence for effects of BAT.

\section{ACKNOWLEDGEMENT}

This work was supported by the 2011 Inje University research grant.

\section{REFERENCES}

1) Feys HM, De Weerdt WJ, Selz BE, et al.: Effect of a therapeutic intervention for the hemiplegic upper limb in the acute phase after stroke. A singleblind, randomized, controlled multicenter trial. Stroke, 1998, 29: 785-792. [Medline] [CrossRef]

2) Sun HS, Park GA, Sohn SJ, et al.: The relationship between hemiplegic upper extremity function and activities of daily living according to age in stroke patients. J Korean Geriatr Soc, 2011, 15: 29-36. [CrossRef]

3) Kilbreath SL, Heard RC: Frequency of hand use in healthy older persons. Aust J Physiother, 2005, 51: 119-122. [Medline] [CrossRef]

4) Debaere F, Wenderoth N, Sunaert S, et al.: Cerebellar and premotor function in bimanual coordination: parametric neural responses to spatiotemporal complexity and cycling frequency. Neuroimage, 2004, 21: 14161427. [Medline] [CrossRef]

5) Duque J, Mazzocchio R, Dambrosia J, et al.: Kinematically specific interhemispheric inhibition operating in the process of generation of a voluntary movement. Cereb Cortex, 2005, 15: 588-593. [Medline] [CrossRef]

6) Stinear JW, Byblow WD: Disinhibition in the human motor cortex is enhanced by synchronous upper limb movements. J Physiol, 2002, 543: 307-316. [Medline] [CrossRef]

7) Yun SY, Lee TY, Park SY, et al.: Muscle activity and a kinematic analysis of drinking motion. J Korean Soc Occup Ther, 2008, 16: 77-88.

8) Uswatte G, Taub E, Morris D, et al.: Reliability and validity of the up- 
per-extremity Motor Activity Log-14 for measuring real-world arm use Stroke, 2005, 36: 2493-2496. [Medline] [CrossRef]

9) Carswell A, McColl MA, Baptiste S, et al.: The Canadian occupational performance measure: a research and clinical literature review. Can J Occup Ther, 2004, 71: 210-222. [Medline]

10) Senesac CR, Davis S, Richards L: Generalization of a modified form of repetitive rhythmic bilateral training in stroke. Hum Mov Sci, 2010, 29: 137-148. [Medline] [CrossRef]

11) McCombe Waller S, Liu W, Whitall J: Temporal and spatial control following bilateral versus unilateral training. Hum Mov Sci, 2008, 27: 749-758 [Medline] [CrossRef]

12) Cauraugh JH, Kim SB, Duley A: Coupled bilateral movements and active neuromuscular stimulation: intralimb transfer evidence during bimanual aiming. Neurosci Lett, 2005, 382: 39-44. [Medline] [CrossRef]
13) Summers JJ, Kagerer FA, Garry MI, et al.: Bilateral and unilateral movement training on upper limb function in chronic stroke patients: a TMS study. J Neurol Sci, 2007, 252: 76-82. [Medline] [CrossRef]

14) Chang JJ, Tung WL, Wu WL, et al.: Effects of robot-aided bilateral force induced isokinetic arm training combined with conventional rehabilitation on arm motor function in patients with chronic stroke. Arch Phys Med Rehabil, 2007, 88: 1332-1338. [Medline] [CrossRef]

15) Rose DK, Winstein CJ: The co-ordination of bimanual rapid aiming movements following stroke. Clin Rehabil, 2005, 19: 452-462. [Medline] [CrossRef]

16) $\mathrm{Wu} \mathrm{CY}$, Hsieh $\mathrm{YW}$, Lin $\mathrm{KC}$, et al.: Brain reorganization after bilateral arm training and distributed constraint-induced therapy in stroke patients: a preliminary functional magnetic resonance imaging study. Chang Gung Med J, 2010, 33: 628-638. [Medline] 